\title{
Imaging the microscopic structure of shear thinning and thickening colloidal suspensions
}

\author{
Xiang Cheng ${ }^{1}$, Jonathan H. McCoy ${ }^{2}$, Jacob N. Israelachvilii and Itai Cohen ${ }^{1}$ \\ ${ }^{1}$ Department of Physics, Cornell University, Ithaca, New York 14853, USA \\ ${ }^{2}$ Department of Physics and Astronomy, Colby College, Waterville, Maine 04901, USA \\ ${ }^{3}$ Materials Research Laboratory, and Department of Chemical Engineering, University of \\ California, Santa Barbara, California 93106, USA
}

\begin{abstract}
The viscosity of colloidal suspensions can vary by orders of magnitude depending on how quickly they are sheared. Although this non-Newtonian behavior is believed to arise from the arrangement of suspended particles and their mutual interactions, microscopic particle dynamics in such suspensions are difficult to measure directly. Here, by combining fast confocal microscopy with simultaneous force measurements, we systematically investigate a suspension's structure as it transitions through regimes of different flow signatures. Our measurements of the microscopic single-particle dynamics unambiguously show that shear thinning results from the decreased relative contribution of entropic forces and that shear thickening arises from particle clustering induced by inter-particle hydrodynamic lubrication forces. This combination of techniques illustrates an approach that complements current methods for determining the microscopic origins of non-Newtonian flow behavior in complex fluids.
\end{abstract}


Non-Newtonian flow phenomena such as the shear thickening of cornstarch and water mixtures, have attracted the interest of scientists, due to their importance for many natural and industrial processes (1-4), as well as that of the general public, as exemplified by the popularity of videos showing people running across swimming pools filled with such fluids (5). As a simple model system, a colloidal suspension of hard spheres in a Newtonian fluid captures the essential features of many non-Newtonian behaviors, including shear thinning and thickening $(1,5-8)$. Pioneering numerical simulations and experiments combining rheology with various scattering techniques have done much to illuminate the microstructural origins of such phenomena (5-15). Nevertheless, numerous fundamental questions remain unresolved (13). For example, although the shear thinning of suspensions at low shear rates is typically associated with the formation of particle layers $(5,6,8,14)$, whether such layering is a major driving force for shear thinning remains unresolved $(6,15)$. As another example, it remains controversial whether shear thickening in dilute suspensions arises from an order-to-disorder transition $(14,15)$, the formation of particle clusters induced by lubrication hydrodynamics $(5,9,10,12,13)$, or the confinement and dilation of suspened particles under shear (18-20).

In part, the difficulty in resolving these questions results from an inability of previous techniques to directly access single-particle dynamics over a range of intermediate length scales. Specifically, exact Stokesian dynamics simulations have typically studied small systems in three dimensions (3D) and have therefore only described local particle dynamics (9), whereas scattering experiments probed very large sample volumes and reported only on average suspension structures (11-13). Hence, there is a need for complementary experimental measurements that bridge these two limits by exploring relatively large volumes with single particle resolution, and that are capable of distinguishing between the various proposed models. Here, by combining fast confocal microscopy $(21,22)$ with simultaneous rheological measurements $(21,23)$, we systematically correlate the real-space microstructure of concentrated hard-sphere suspensions with their flow properties.

Our samples consist of silica spheres with diameter $2 a=0.96 \mu \mathrm{m}$ and polydispersity of $5 \%$.

Particles are suspended in a water-glycerin mixture with matching index of refraction and solvent viscosity $\eta_{0}=0.06 \mathrm{~Pa} \cdot \mathrm{s}$. Fluorescein sodium salt is added to the solvent so that particles appear as dark dots on a bright background. For the experimentally relevant volume fractions, $0.30 \leq \phi<$ 0.48 , the suspension is in the fluid state at equilibrium, which is consistent with the phase 
diagram of hard sphere suspensions. Thus, under shear, the suspension properties are determined solely by entropic and hydrodynamic forces.

Our shear cell mounts on a high speed confocal microscope, which allows for direct imaging of the 3D suspension structure (Fig. 1A) (24). The shear cell consists of two parallel plates: a movable microscope cover slip as the bottom plate and a fixed $16 \mathrm{~mm}^{2}$ square silicon wafer as the top plate. Both plates are flat on the particle length scale. Using set screws the plates can be made parallel within $0.0075^{\circ}$. In our experiments, we use a plate separation of $h=6.4 \pm 0.3 \mu \mathrm{m}$, which allows us to reproduce the bulk suspension rheology and rapidly scan the entire sample in $3 \mathrm{D}$. A piezoelectric actuator generates sinusoidal motion of the bottom plate, $x(t)=A \sin (2 \pi f t)$, with amplitude $A=22 \mu \mathrm{m}$ and frequency $0 \leq f \leq 100 \mathrm{~Hz}$. Thus, our experiments are conducted at shear strain $\gamma \equiv A / h=3.67$ and at shear rates $\dot{\gamma}_{0}=2 \pi f(A / h)$ up to 2000. The corresponding Pelect number $\mathrm{Pe} \equiv \eta_{0} \dot{\gamma}_{0} a^{3} / k_{B} T$, the dimensionless ratio comparing particle advection to diffusion, is 3200 at the maximum shear rate. To measure the 3D structure, a stack of images oriented parallel to the shear velocity - vorticity plane $(x-z)$ is taken along the velocity gradient direction (y) (Fig. 1B). Finally, the upper plate is attached to a custom load cell that measures the shear stress. Collectively, this apparatus functions as a "confocal rheoscope" $(21,23)$.

Using this apparatus, we measure the frequency dependence of the dynamic shear viscosity, $\eta \equiv \tau_{0} / \dot{\gamma}_{0}$, and the phase lag between stress and strain, $\delta$. Here, $\tau_{0}$ is the sinusoidal shear stress amplitude. These material properties are plotted versus Pe for two suspensions with $\phi=$ $0.34 \pm 0.03$ and $0.47 \pm 0.03$ in Fig. 1C. The data show regimes corresponding to shear thinning, Newtonian, and shear thickening behavior. In the shear thinning regime where $\mathrm{Pe} \leq 3.6, \delta$ is smaller than $\pi / 2$, indicating the samples are viscoelastic. As Pe increases beyond 3.6, $\delta$ is nearly $\pi / 2$, signifying a purely viscous response. In this regime $\eta$ remains constant and the suspensions behave as Newtonian fluids. For $\mathrm{Pe} \geq 167$, the high $\phi$ sample shear thickens and becomes more elastic as indicated by a slight decrease in $\delta$. Although we cannot resolve clear shear thickening in the low $\phi$ sample, our measurements are consistent with bulk rheometer studies where very weak thickening is observed (13). Our data over the entire range of Pe quantitatively reproduce rheological measurements of bulk suspensions $(5,8,13)$. 
These rheological transitions correlate with changes in particle configurations. To quantify the suspension structure, we generate a real-time 3D pair correlation function $g(\vec{r})$ from low to intermediate $\mathrm{Pe}$, which is defined as the probability of finding a particle at position $\vec{r}$ with respect to each particle center. We plot 2D cuts of $g(\vec{r})$ along the three orthogonal planes centered at the origin, as shown in Fig. 1, D-G. At $\mathrm{Pe}=0.036$, the particle configuration is isotropic in the $x-z$ plane as indicated by bright rings representing the first and second shells of neighboring particles (Fig. 1D). In the $y-z$ and $x-y$ planes, we observe a slight anisotropy due to particle layering induced by the shear plate confinement (Fig. 1, E and F) (25). More importantly, these observed patterns remain nearly constant throughout the oscillation cycle (movie S1). In contrast, with increasing Pe, we find that while $g(x, z)$ and $g(y, z)$ remain constant, $g(x, y)$ begins to exhibit strong oscillatory distortions as the suspension shear thins (Fig. 2, A-D, movie S2, S3). Simultaneously, we also observe the sharpening of horizontal bands in $g(x, y)$ and $g(y, z)$ (Fig. 1, E and F, and Fig. 2, A-D, movies S1-S3).

These structural signatures illustrate both the increase of particle layering and the changing contribution of entropic stress to the total stress during shear thinning. The enhancement of particle layers is thought to decrease the suspension viscosity by lowering dissipation due to collisions between particles $(6,8,14,15)$. In our experiments, layering is indicated by the sharpening of horizontal bands in $g(x, y)$ and $g(y, z)$ (Fig. 2, A-D) and can be quantified by measuring the number density of particles along the $y$ direction, $\rho(y)$, for different Pe (Fig. 2E). To track the degree of layering in our suspension, we define the order parameter, $\xi(\mathrm{Pe})=\frac{1}{N} \sum_{i=1}^{N} \frac{\rho_{i}(\mathrm{Pe})}{\rho_{i}(\mathrm{Pe}=0)}$, where $\rho_{i}$ is the height of the $i$ th peak relative to its adjacent valley in $\rho(y)$ and $N$ corresponds to the number of layers. We find that $\xi$ increases throughout the shear thinning regime, plateaus when the suspension transitions to the Newtonian regime, and decays prior to the onset of shear thickening (Fig. 2F). Additionally, we observe that as the suspension layers, particles form log-rolling strings aligned along the vorticity direction within the layers [supporting online material (SOM), Fig. S2].

The increase and decrease in layering and in-layer structure is comparable at low and high Pe (Fig. $2 \mathrm{~F}$, Fig. S2F). However, the magnitude of the decrease in $\eta$ during shear thinning is much larger than the increase in $\eta$ during shear thickening (Fig. 1C). This asymmetry indicates layering does 
not account for the measured large viscosity changes and points to an entropic origin for shear thinning $(5,6,8,9)$.

The entropic contribution to the stresses can be determined from distortions induced in $g(\vec{r})$ during shear. At low Pe, the relatively small distortions in $g(\vec{r})$ indicates Brownian motion is sufficiently rapid to restore the equilibrium suspension structure from the entropically less favorable shear-induced configurations. At higher Pe, the observed distortions reflect the decreased contribution of the entropic stresses relative to hydrodynamic stresses induced by the applied shear. Under the influence of the hydrodynamic stresses, particles are squeezed together along the $45^{\circ}$ major compressive axis and are separated out along the $135^{\circ}$ major extensional axis (Fig. 2, B and D, movies S2, S3) $(26,27)$. We quantify the degree of the distortion at different Pe by performing a $3 \mathrm{D}$ integral around the first peak of $g(\vec{r})$, which determines the entropic contribution to the stresses (Fig. 3A)(SOM text). We show quantitatively that in the shear thinning regime, the entropic contributions to the total viscosity decrease monotonically (Fig. 3B blue circles). We assume that the hydrodynamic contribution to the viscosity is constant at low Pe and can be determined from the plateau viscosity in the Newtonian regime (Fig. 1C). Remarkably, we find that adding the constant hydrodynamic viscosity (Fig. 3B dashed line) to the entropic viscosity completely accounts for the macroscopically measured shear thinning data in Fig. 1C (Fig. 3B blue disks). These entropic stresses also lead to the viscoelastic flow properties of the suspension during shear thinning (SOM text). Since the entropic stresses result from distortions in the equilibrium structure of the suspension, the only way that layering can affect shear thinning is if the equilibrium structure is already layered, an effect that can for example arise from further confinement.

At higher Pe, the contributions of entropy to the suspension viscosity become negligible. Our data further indicates that changes in the layering and in-layer order do not alter the suspension viscosity in the shear thinning and Newtonian regimes. Therefore, we expect they do not contribute to shear thickening either. Consistent with this interpretation, we find that the decrease in layering and in-layer structure are not correlated with the onset of shear thickening (Fig. 2F, Fig. S2F). Furthermore, since the volume fractions of our suspensions are well below that for the jamming or glass transition, the system is dominated by hydrodynamic interactions rather than frictional contact between particles (18-20). These observations are consistent with the prediction 
that hydroclusters - groups of particles whose relative motions are restricted by lubrication stresses - are responsible for shear thickening $(5,9,10)$.

Enhanced hydrodynamic coupling between particles in the shear thickening regime can be directly observed in our confocal movies by studying the motions of particles in adjacent layers (movie S4, S5, S6 and SOM text). To identify the hydroclusters, we perform a cluster analysis on our data. We define a threshold interparticle distance $D$ for including a particle in a cluster, and determine the probability $P_{N}$ of obtaining a cluster with $N$ particles. $D$ is chosen to be the largest distance that leads to an exponential decay in $P_{N}$ for stationary suspensions, and its value is roughly one particle diameter (SOM text). We find that the exponential decay of $P_{N}$ persists until the onset of shear thickening (Fig. 4A). Once the suspension begins to shear thicken however, we measure a greater probability for obtaining large clusters. Furthermore, we find that neighboring particles in the clusters preferentially align along the $45^{\circ}$ and $135^{\circ}$ in the flow-gradient $(x-y)$ plane (Fig. 4B) and along the flow direction in the flow-vorticity $(x-z)$ plane (Fig. 4C). These cluster morphologies are consistent with those predicted by numerical simulations (28) and account for the slight elastic response. Thus, using these techniques we directly identify and visualize hydroclusters as the origin of shear thickening in colloidal suspensions (Fig. 4D).

Collectively, our measurements over the entire range of Pe unambiguously demonstrate the coupling between microstructure and macroscopic flow behaviors in colloidal suspensions. They provide crucial experimental evidence for determining the underlying physics behind shear thinning and thickening. The combination of imaging techniques and force measurements presented illustrates a new approach for investigating the microscopic origins of non-Newtonian flow behavior in various structured fluids. For example, changing the morphology of the shearing surfaces and degree of confinement will enable investigation of phenomena relevant for lubrication and bio-rheology (29). 


\section{References and Notes}

(1) R. G. Larson, The Structure and Rheology of Complex Fluids (Oxford Univ. Press, New York, 1999).

(2) D. L. Turcotte, G. Schubert, Geodynamics (Cambridge University Press; 2 edition, Cambridge UK, 2001).

(3) Y. Dzenis, Science 304, 1917 (2004).

(4) P. Beiersdorfer, D. Layne, E. W. Magee, J. I. Katz, Phys. Rev. Lett. 106, 058301 (2011).

(5) N. J. Wagner, J. F. Brady, Phys. Today 62, 27 (2009).

(6) J. J. Stickel, R. L. Powell, Annu. Rev. Fluid Mech. 37, 129 (2005).

(7) J. Vermant, M. J. Solomon, J. Phys: Condens. Matter 17, R187 (2005).

(8) J. M. Brader, J. Phys.: Condens. Matter 22, 363101 (2010).

(9) D. R. Foss, J. F. Brady, J. Fluid Mech. 407, 167 (2000).

(10) J. R. Melrose, R. C. Ball, J. Rheol. 48, 961 (2004).

(11) H. M. Laun et al., J. Rheol. 36, 743 (1992).

(12) B. J. Maranzano, N. J. Wagner, J. Chem. Phys. 117, 10291 (2002).

(13) D. Kalman, N. J. Wagner, Rheol. Acta. 48, 897 (2009).

(14) R. L. Hoffman, J. Colloid Interface Sci. 46, 491 (1974).

(15) R. L. Hoffman, J. Rheol. 42, 111 (1998).

(16) J. J. Erpenbeck, Phys. Rev. Lett. 52, 1333 (1984).

(17) J. Delhommelle, J. Petravic, D. J. Evans, Phys. Rev. E 68, 031201 (2003).

(18) A. Fall, N. Huang, F. Bertrand, G. Ovarlez, D. Bonn, Phys. Rev. Lett. 100, 018301 (2008). 
(19) E. Brown et al., Nature Mater. 9, 220 (2010).

(20) E. Brown, H. M. Jaeger, Preprint at $<$ http://arxiv.org/abs/1010.4921> (2010).

(21) L. Isa, R. Besseling, A. B. Schofield, W. C. K. Poon, Preprint at $<$ http://arxiv.org/abs/0907.5440> (2009).

(22) Y. L. Wu, D. Derks, A. van Blaaderen, A. Imhof, Proc. Natl. Acad. Sci. USA 106, 10564 (2009).

(23) K. M. Schmoller, P. Fernández, R. C. Arevalo, D. L. Blair, A. R. Bausch, Nature Commun. 1, 134 (2010).

(24) Materials and methods are available as supporting material on Science Online.

(25) C. R. Nugent, K. V. Edmond, H. N. Patel, E. R. Weeks, Phys. Rev. Lett. 99, 025702 (2007).

(26) F. Parsi, F. Gadala-Maria, J. Rheol. 31, 725 (1987).

(27) C. Gao, S. D. Kulkarni, J. F. Morris, J. F. Gilchrist, Phys. Rev. E 81, 041403 (2010).

(28) J. F. Brady, G. Bossis, J. Fluid. Mech. 155, 105 (1985).

(29) M. Urbakh, J. Klafter, D. Gourdon, J. Israelachvili, Nature 430, 525 (2004).

(30) We thank T. Beatus, Y.-C. Lin, J. Brady, L. Ristroph, and N. Wagner for useful discussions. This research was supported by grants from the NSF CMMI, and in part by award no. KUS-C1-018-02 from King Abdullah University of Science and Technology (KAUST). 


\section{Supporting Online Material}

www.sciencemag.org

Materials and Methods

Figs. S1 to S6

References (31-36) [Note: The numbers refer to any additional references cited only within the $\mathrm{SOM}]$

Movie S1 to S6

\section{Figure captions}

Fig. 1. (A) Schematic of our confocal rheoscope. CM: confocal microscope, SG: strain gauge, CS: colloidal suspension, ST: solvent trap to prevent evaporation, PTS: piezo translation stage. (B) Definition of coordinate system. (C) Rheology of colloidal suspensions versus Pe for $\phi=0.34$ (square) and $\phi=0.47$ (circle). Upper panel shows the magnitude of the dynamic shear viscosity, $\eta$. Lower panel shows the phase angle between stress and strain, $\delta$. Vertical dashed lines mark the end of shear thinning and the onset of shear thickening. (D-G) 3D pair correlation function, $g(\vec{r})$, for $\phi=0.34$ sample at $\mathrm{Pe}=0.036$. (D-F) show the cuts of $g(\vec{r})$ in the $z-x, x-y$ and $z-y$ planes while $(\mathbf{G})$ indicates their relative orientation. Different planes are indicated by different bounding box colors.

Fig. 2. (A-D) show $g(x, y)$ for $\phi=0.34$ sample at $\mathrm{Pe}=0.36$. Each figure corresponds to a specific phase in the oscillation cycle with oscillation angle approximately $\pi / 2(\mathbf{A}), \pi(\mathbf{B}), 3 \pi / 2(\mathbf{C})$ and $2 \pi$ (D). Shear-induced compression and extension at maximum negative and positive shear rates are illustrated by black arrows in (B) and (D). Layer structure is indicated by yellow arrows. (E) Number density of particles, $\rho$ versus $y$ for $\phi=0.34$ sample. $\rho(y)$ indicates the fraction of particles that are located in a slice of thickness $d$ centered at $y$. Curves for different Pe are shifted 
vertically for clarity. (F) Order parameter, $\xi$, versus Pe for $\phi=0.34$ (square) and $\phi=0.48$ (circle) samples.

Fig. 3. (A) Entropic shear stresses, $\sigma_{E}$, calculated from $g(\vec{r})$ for $\phi=0.47$ at shear frequency $f=$ $2 \times 10^{-4} \mathrm{~Hz}$ and $\mathrm{Pe}=0.015$. The period of the shear $T=1 / f=5000 \mathrm{~s}$. The blue line is the fit of $\sigma_{E}$ with a sinusoidal oscillation. The red line is the corresponding shear strain. (B) Shear thinning induced by entropic stresses for $\phi=0.47$. Blue circles show the entropic viscosity $\eta_{E}=\left|\sigma_{E}\right| /|\dot{\gamma}|$. Horizontal dashed line indicates the hydrodynamic viscosity, $\eta_{H}$, determined from the Newtonian plateau in Fig. 1C. Blue disks show the total viscosity $\eta=\eta_{E}+\eta_{H}$. Black circles show the viscosity from macroscopic load cell measurement (Fig. 1C). Red solid line is the fit of the empirical expression for shear thinning $\eta=\eta_{\infty}+\left(\frac{\eta_{0}-\eta_{\infty}}{1+b \mathrm{Pe}}\right)(1)$.

Fig. 4. Hydroclusters for $\phi=0.47$ sample. (A) The probability distribution for cluster size, $P_{N}$, at different Pe. (B and $\mathbf{C}$ ) show the probability distribution of the orientation of neighboring particles in $N \geq 6$ clusters. The orientation is projected into the $x-y$ plane (B) and the $x$-z plane (C) respectively with corresponding angles defined in the insets. Red lines are guides to eye. Results are averaged over a full cycle of shear. (D) shows the instantaneous real-space configuration of hydroclusters with $N \geq 6$ at shear phase 0.7 . Different colors indicate different clusters. Particles outside the large clusters are drawn with smaller size for clarity. The boundary box is $31.2 \times 15.4 \times 3.1 \mu \mathrm{m}^{3}$. 
Figure:
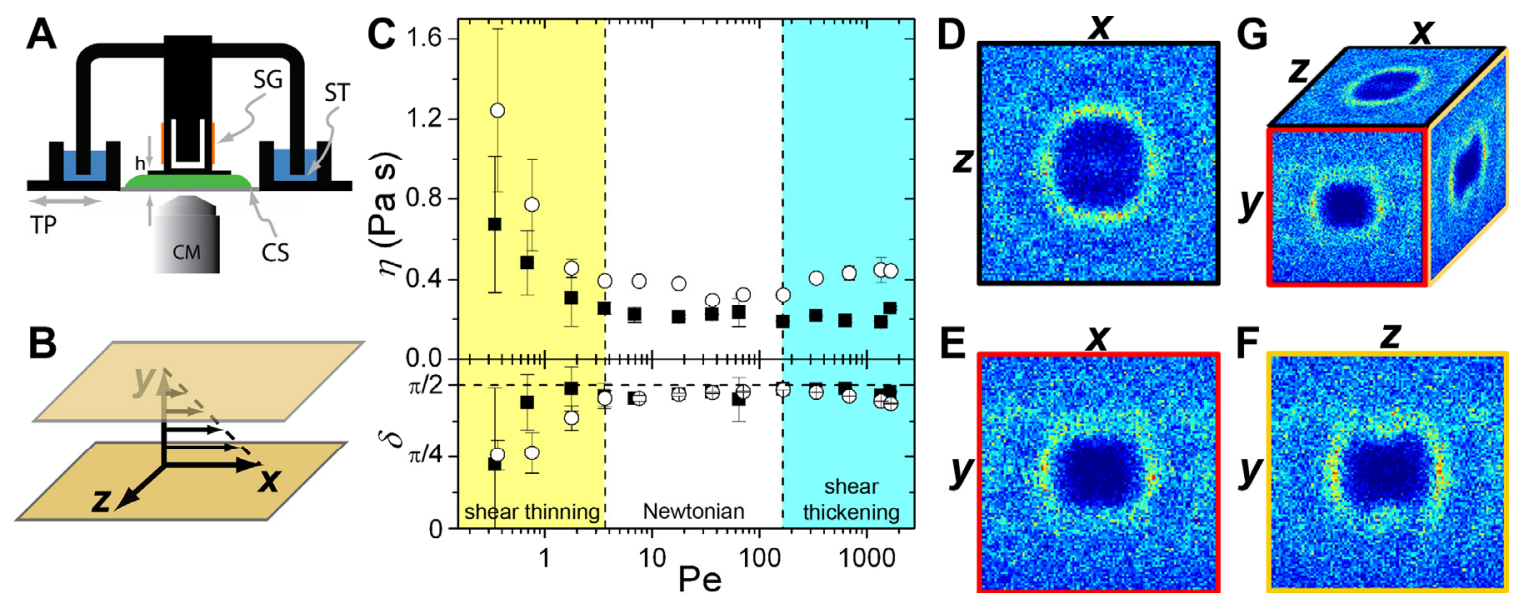

Fig. 1 

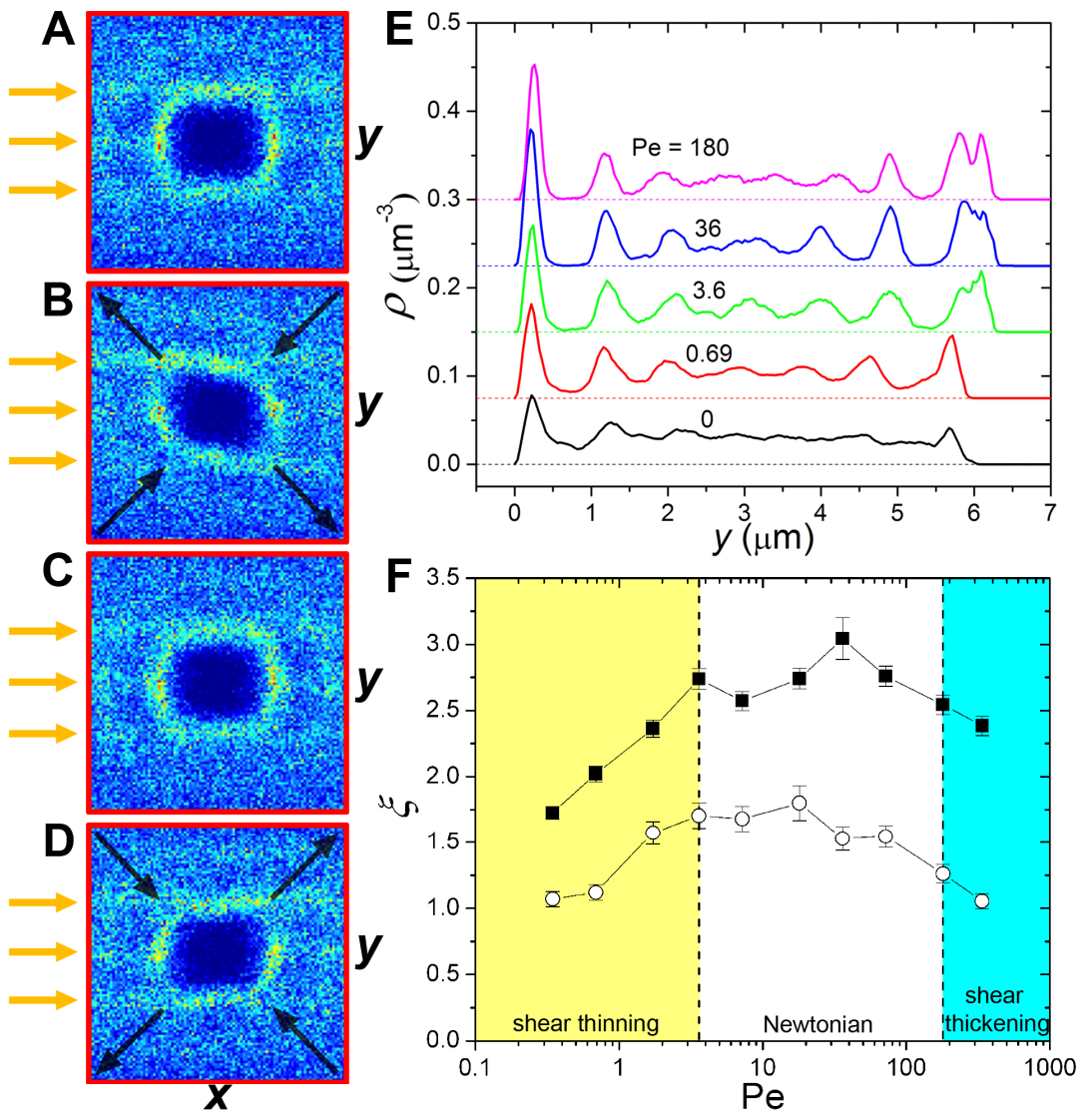

Fig. 2 

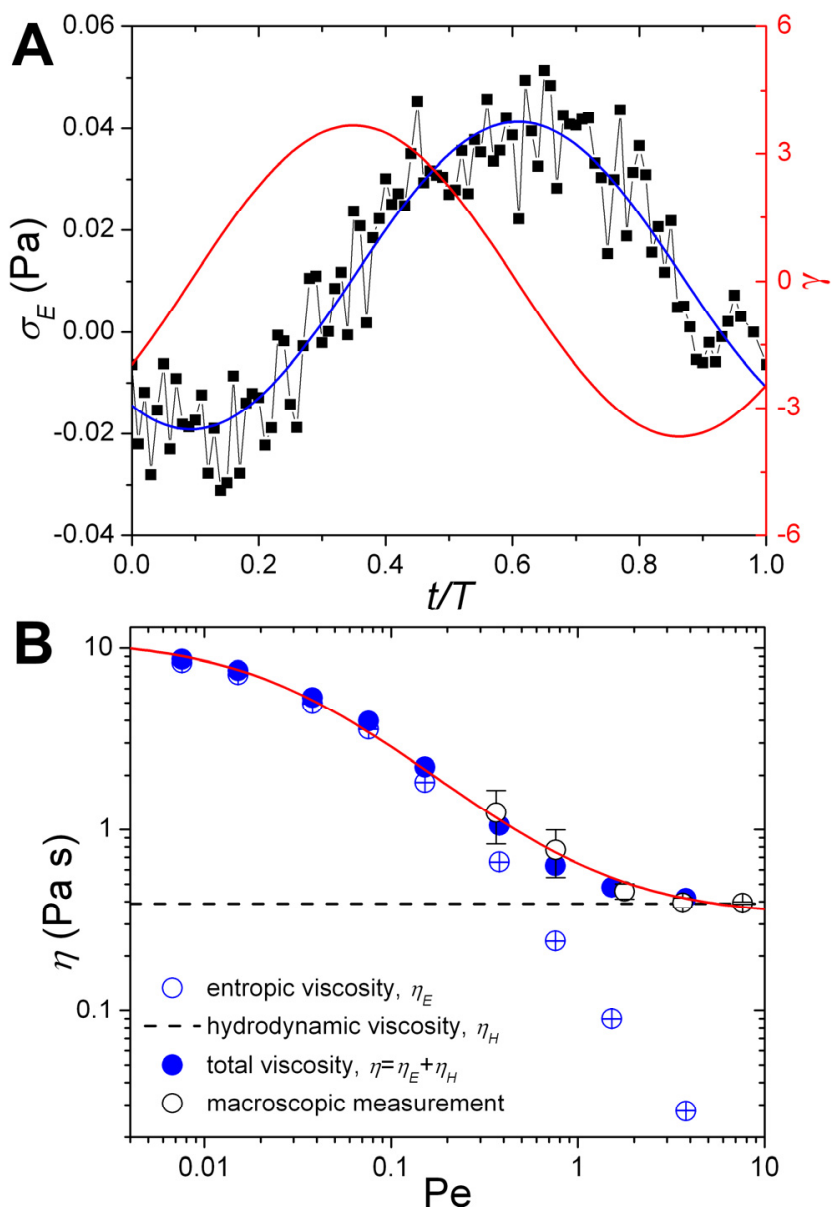

Fig. 3 

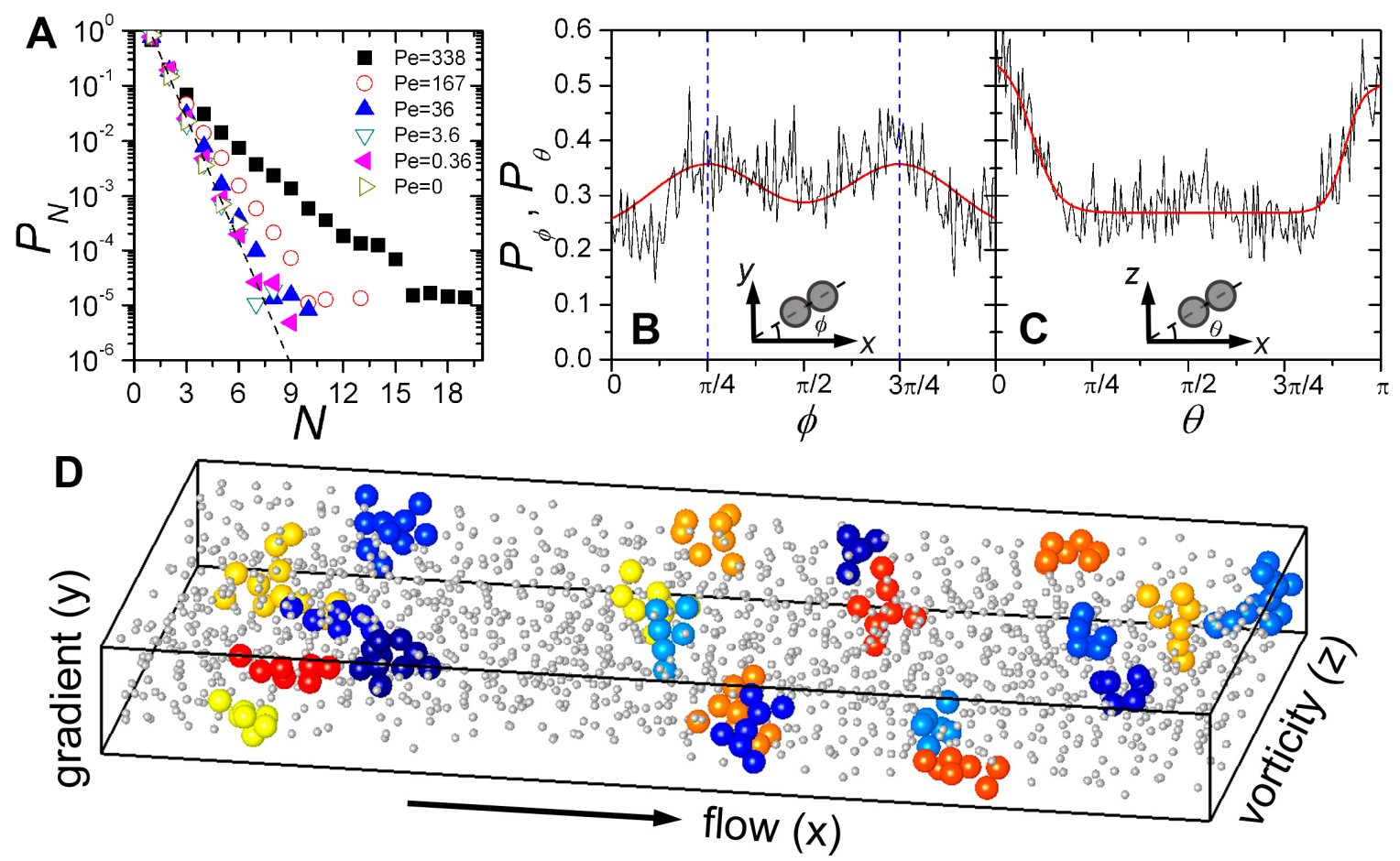

Fig. 4 